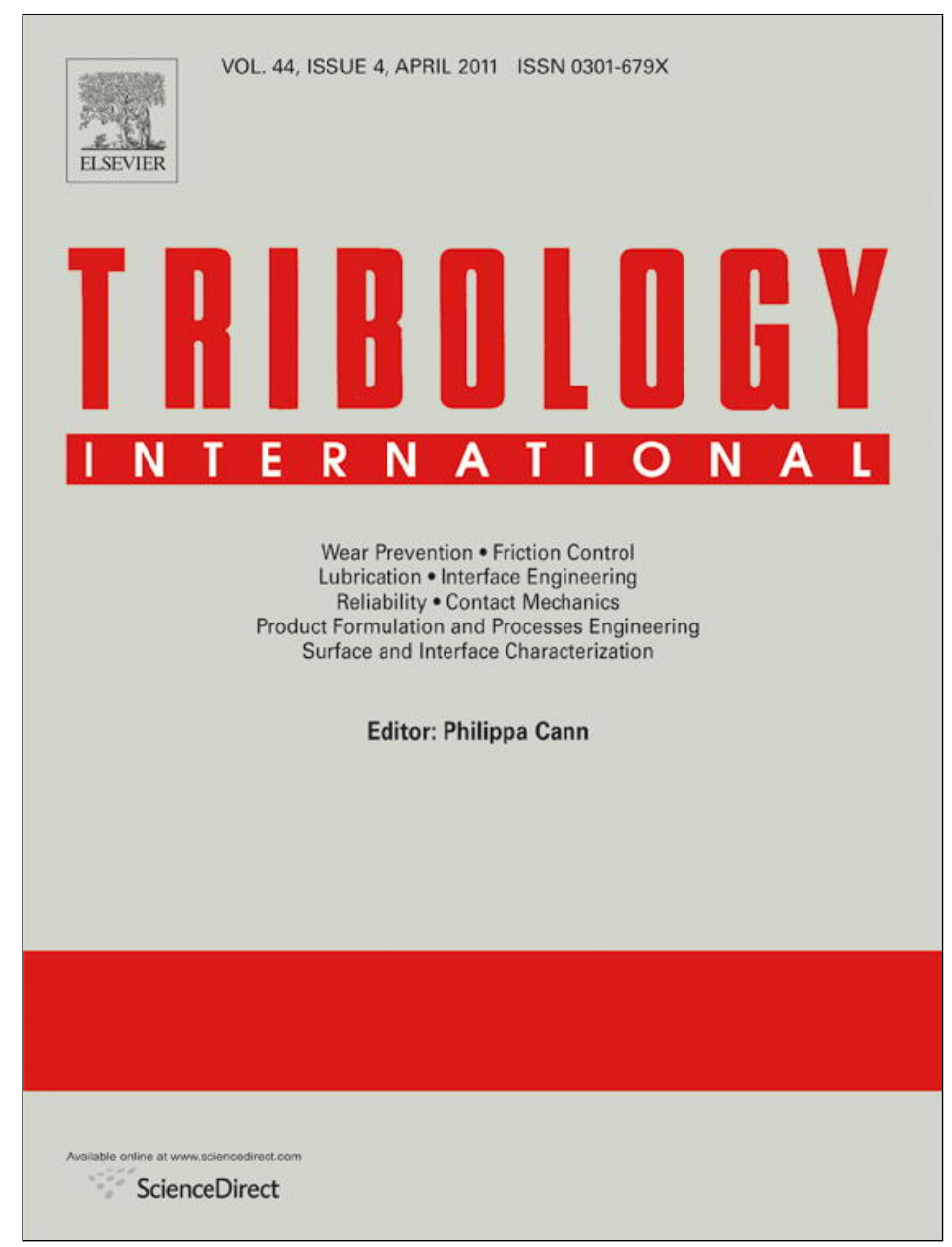

This article appeared in a journal published by Elsevier. The attached copy is furnished to the author for internal non-commercial research and education use, including for instruction at the authors institution and sharing with colleagues.

Other uses, including reproduction and distribution, or selling or licensing copies, or posting to personal, institutional or third party websites are prohibited.

In most cases authors are permitted to post their version of the article (e.g. in Word or Tex form) to their personal website or institutional repository. Authors requiring further information regarding Elsevier's archiving and manuscript policies are encouraged to visit:

http://www.elsevier.com/copyright 


\title{
Thermodynamics and kinetics of boundary friction
}

\author{
I.A. Lyashenko ${ }^{\text {a,* }}$, A.V. Khomenko ${ }^{\text {a }}$, L.S. Metlov ${ }^{\text {b }}$ \\ a Sumy State University, 40007 Sumy, Ukraine \\ ${ }^{\mathrm{b}}$ Donetsk Institute for Physics and Engineering named after O.O. Galkin of the NASU, 83114 Donetsk, Ukraine
}

\section{A R T I C L E I N F O}

Article history:

Received 5 July 2010

Received in revised form

23 November 2010

Accepted 14 December 2010

Available online 21 December 2010

Keywords:

Ultrathin lubricant film

Friction force

Phase transition

Stick-slip motion

\begin{abstract}
A B S T R A C T
A deterministic theory describing the behavior of an ultrathin lubricant film between two atomically smooth solid surfaces is proposed. For the description of lubricant state the parameter of excess volume arising due to chaotization of solid medium structure in the course of melting is introduced. Thermodynamic and shear melting are described. Dependences of friction force on temperature of lubricant, shear velocity of rubbing surfaces, and pressure upon surfaces are analyzed. Within the framework of a simple tribological model the stick-slip mode of friction, when the lubricant periodically melts and solidifies, is described. The obtained results are qualitatively compared with the experimental data.
\end{abstract}

(c) 2010 Elsevier Ltd. All rights reserved.

\section{Introduction}

The boundary friction mode arising with lubricant thickness less than 10 atomic layers is widely investigated so far [1]. Experiments show that a thin layer of lubricant shows anomalous properties compared with volume lubricants [2,3]. In particular, the intermittent motion (stick-slip) inherent in a dry friction is observed [2-4]. Such mode is explained as the solidification caused by compression of rubbing surfaces followed by jump-like melting at a shear stress larger than the yield limit ("shear melting").

There are some phenomenological models, for example, thermodynamic [5], mechanistic [6] and synergetic [7] ones, allowing to explain the experimental results partially. They are either deterministic [6,7] or stochastic [8,9] in nature. Studies by using molecular dynamics methods are also known [10-13]. It turns out that the lubricant provides several kinetic modes with transitions leading to the stick-slip friction $[2,3]$. Such transitions are the first-order phase transitions [14], however, between the states which are not true thermodynamic phases but the kinetic modes of friction. In work [8] three modes are found: sliding at low shear velocities, regular stickslip mode, and sliding at the high shear velocities. Their existence has been verified in numerous experiments $[1-4,15]$.

In work [7], within the framework of Lorenz model, to approximate viscoelastic medium, an approach has been developed according to which the transition of ultrathin lubricant film from solid- into liquid-like state results from the thermodynamic and shear melting. The processes due to self-organization of shear stress and strain fields

\footnotetext{
* Corresponding author.

E-mail address: nabla04@ukr.net (I.A. Lyashenko).
}

and of lubricant temperature are described in view of additive noises of the specified quantities [16-19] and correlated temperature fluctuations [20]. In works $[18,19]$ within the framework of this model the multifractal behavior of stress time series is investigated. Many physical objects have multifractal characteristics. For example, this modern direction in physics includes the investigation of fatigue process $[21,22]$. The melting of lubricant film under friction between atomically flat surfaces at different temperature dependences of viscosity is described in [23]. The reasons for jump-like melting and hysteresis, observed experimentally [24-26], are considered in works $[27,28]$. Here, realization conditions of these features are determined with the deformation defect of the shear modulus taken into account. In work [29], within the framework of the mentioned model, the periodic stick-slip mode of friction is described which, however, has a stochastic component and can be realized only in the presence of fluctuations. The drawback of the model is that it does not consider the load applied to the surfaces of friction and that basic equations are derived by several approximations in [7].

Existence of a considerable quantity of approaches to the solution of the considered problem is certainly caused by its complexity. Methods of molecular dynamics [10-13] give good results, however, in this case, to describe each concrete experimental situation it is necessary to derive various systems of equations and it is therefore difficult to track a general tendency. Microscopic theories often cannot explain the behavior of macroscopic quantities which are actually measured in experiments. Besides, with computer modeling it is often impossible to describe long-time processes as a result of computing restrictions. The phenomenological approach, in particular, shown in this work, gives a chance to bypass the specified difficulties and can help in connecting the parameters of microscopic theories with macroscopic measurements. In Ref. [30] it is noted that to describe 


\begin{tabular}{|c|c|c|c|}
\hline \multicolumn{2}{|c|}{ Nomenclature } & \multirow{2}{*}{$\begin{array}{l}\sigma_{i j} \\
\sigma_{i j}^{\nu i s c} \\
F_{i j}, F\end{array}$} & \multirow{2}{*}{$\begin{array}{l}\text { total tensor of stress }(\mathrm{Pa}) \\
\text { tensor of viscous components of stress }(\mathrm{Pa}) \\
\text { friction force }(\mathrm{N})\end{array}$} \\
\hline$\Phi$ & density of free energy $\left(\mathrm{J} / \mathrm{m}^{3}\right)$ & & \\
\hline$f$ & excess volume (dimensionless variable) & $A$ & area of contact of rubbing surfaces $\left(\mathrm{m}^{2}\right)$ \\
\hline$T$ & temperature of lubricant $(\mathrm{K})$ & $\eta_{\text {eff }}$ & effective viscosity of lubricant (Pa s) \\
\hline & tensor of elastic strain (dimensionless variable) & $\gamma$ & phenomenological coefficient (dimensionless \\
\hline & first invariant of strain tensor (dimensionless variable) & & \\
\hline$\left(\varepsilon_{i j}^{e}\right)^{2}$ & second invariant of strain tensor (dimensionless & $k$ & proportionality coefficient $\left(\mathrm{Pa} \mathrm{s}^{\gamma+1}\right.$ ) \\
\hline & variable) & $L$ & load on the top surface of friction $(\mathrm{N})$ \\
\hline$\sigma_{i j}^{e}$ & tensor of elastic stress $(\mathrm{Pa})$ & $T_{c 0}$ & temperature of melting of lubricant $(\mathrm{K})$ \\
\hline$\mu_{\text {eff }}, \lambda_{\text {eff }}$ & effective elastic constants (Pa) & $T_{c}^{0}$ & temperature of solidification of lubricant $(\mathrm{K})$ \\
\hline & normal component of stresses $(\mathrm{Pa})$ & $V_{c 0}$ & critical velocity of rubbing block for melting of \\
\hline$\tau$ & shear component of stresses $(\mathrm{Pa})$ & & lubricant $(\mathrm{m} / \mathrm{s})$ \\
\hline $\begin{array}{c}\tau_{f} \\
\varepsilon_{i:}^{p l}\end{array}$ & $\begin{array}{l}\text { relaxation time for excess volume (Pa s) } \\
\text { tensor of plastic strain (dimensionless variable) }\end{array}$ & $V_{c}^{0}$ & $\begin{array}{l}\text { critical velocity of rubbing block for solidification of } \\
\text { lubricant }(\mathrm{m} / \mathrm{s})\end{array}$ \\
\hline${ }^{{ }^{i} i j}$ & Maxwell relaxation time of internal stress (s) & $X$ & coordinate of rubbing block (m) \\
\hline & total tensor of strain (dimensionless variable) & $M$ & mass of rubbing block $(\mathrm{kg})$ \\
\hline$V_{i j}, V$ & velocity of shear of rubbing block $(\mathrm{m} / \mathrm{s})$ & $K$ & rigidity of spring $(\mathrm{N} / \mathrm{m})$ \\
\hline & thickness of lubricant (m) & $V_{0}$ & velocity of free part of spring $(\mathrm{m} / \mathrm{s})$ \\
\hline & stationary value of tensor of elastic strain (dimension- & $\Delta X$ & value of extension of a spring $(\mathrm{m})$ \\
\hline & less variable) & $t, t^{\prime}$ & time of friction process $(\mathrm{s})$ \\
\hline
\end{tabular}

the behavior of contacts in model of earthquakes the effects of plastic deformation need be considered. This is obviously possible within the framework of our approach which includes rheological parameters. In work [31] the formal order parameter is used. It is physically connected with density of dislocations, but it is introduced purely formally it is equal to 0 in one phase and to 1 in another. After that the free energy is chosen with extrema at these values of parameters. An important, in particular for our study, step is taken in [32] for explanation of the stick-slip friction using free volume as a slowly relaxing state parameter.

In the present work, the theory is proposed based on the expansion of free energy of the system into a power series of parameter $f$ which, being squared, represents the excess volume arising from the formation of defect structure in the lubricant during melting. In the loading diagram the liquid-like state is usually interpreted as a section of plastic flow and characterized by the presence of defects in the lubricant [5]. Constructing our model we start with the first principles of thermodynamics. Here, the approach based on the modified Landau theory of phase transitions $[33,34]$ is used to describe strongly nonequilibrium processes, occurring at sliding of two rubbing solid surfaces separated by a layer of lubricant. In this paper we are trying to represent the stick-slip mode of the boundary friction using the thermodynamic model [35]. But here the entropy factors and nonhomogenous effects are not considered. Thus, the suggested model is simpler than that of [35], but the basic results are similar.

\section{Basic equations}

The melting of lubricants whose thickness is less than 10 molecular layers is judged by the increase in their volume [10] and diffusion factor $[10,11,36,37]$. Since, of the two, the experimentally observable quantity is the volume, to describe the lubricant state we introduce parameter $f$, which, when squared, stands for the excess volume arising due to chaotization of structure of a solid medium under melting. With the increase in $f$ magnitude the density of defects in the lubricant is growing, and owing to their transport under the applied stresses the lubricant passes to a kinetic mode of plastic flow (liquid-like phase). Our "order parameter" is precisely connected with excess volume of a liquid-like phase over a solid-like phase, and with its respective excess energy. It can be measured. At small values this redundance is connected with dislocations, at approach to a liquid-like phase it is a chaotically fluctuating density of medium inherent in a liquid phase. Within the framework of this work we use the symmetric model in which the excess volume takes zero value in the solid-like phase of lubricant. Therefore, our approach is more suitable for the description of behavior of lubricant with spherical molecules, when all the atoms are closely packed in the solid-like structure. The excess volume is different from zero in the liquid-like state, it has a larger value at the high temperature of lubricant. When lubricant solidifies, the symmetry of state decreases. Thus, this parameter, as the order parameter, describes the symmetry change at transition.

Let us write down the dependence of the density of free energy $\Phi$ on excess volume in the form of expansion in terms of parameter $f$ [35]

$\Phi=\Phi_{0}+\frac{c}{2}(\nabla f)^{2}+\varphi_{0} f^{2}-\frac{1}{4} \varphi_{1} f^{4}+\frac{1}{6} \varphi_{2} f^{6}$,

where $\Phi_{0}, c, \varphi_{0}, \varphi_{1}, \varphi_{2}$ are expansion constants.

We consider the dependences of elastic strains $\varepsilon_{i j}^{e}$ and lubricant temperature $T$ on invariants for the smaller expansion powers only

$\Phi_{0}=\Phi_{0}^{*}+\frac{1}{2} \lambda\left(\varepsilon_{i i}^{e}\right)^{2}+\mu\left(\varepsilon_{i j}^{e}\right)^{2}$,

$\varphi_{0}=\varphi_{0}^{*}-\frac{1}{2} \bar{\lambda}\left(\varepsilon_{i i}^{e}\right)^{2}-\bar{\mu}\left(\varepsilon_{i j}^{e}\right)^{2}-\alpha T$.

The first invariant represents the trace of strain tensor $\varepsilon_{i i}^{e}=\varepsilon_{1}^{e}+\varepsilon_{2}^{e}+\varepsilon_{3}^{e}$ and the second one is defined by the expression [38]

$$
\begin{aligned}
\left(\varepsilon_{i j}^{e}\right)^{2} & \equiv\left(\varepsilon_{l l}^{e}\right)^{2}-2 I_{2}=\left(\varepsilon_{1}^{e}+\varepsilon_{2}^{e}+\varepsilon_{3}^{e}\right)^{2}-2\left(\varepsilon_{1}^{e} \varepsilon_{2}^{e}+\varepsilon_{1}^{e} \varepsilon_{3}^{e}+\varepsilon_{2}^{e} \varepsilon_{3}^{e}\right) \\
& =\left(\varepsilon_{1}^{e}\right)^{2}+\left(\varepsilon_{2}^{e}\right)^{2}+\left(\varepsilon_{3}^{e}\right)^{2} .
\end{aligned}
$$

According to (1), in the lubricant the elastic stresses appear

$\sigma_{i j}^{e}=\frac{\partial \Phi}{\partial \varepsilon_{i j}^{e}}=\lambda \varepsilon_{i i}^{e} \delta_{i j}+2 \mu \varepsilon_{i j}^{e}-\left(\bar{\lambda} \varepsilon_{i i}^{e} \delta_{i j}+2 \bar{\mu} \varepsilon_{i j}^{e}\right) f^{2}$.

Eq. (5) can take the form of the effective Hooke law [35,39]

$\sigma_{i j}^{e}=2 \mu_{\text {eff }} \varepsilon_{i j}^{e}+\lambda_{\text {eff }} \varepsilon_{i i}^{e} \delta_{i j}$,

with the effective elastic parameters ${ }^{1}$

$\mu_{\text {eff }}=\mu-\bar{\mu} f^{2}$,

\footnotetext{
${ }^{1}$ For $f^{2}>\mu / \bar{\mu}$, we put $\mu_{\text {eff }}=0$ and for $f^{2}>\lambda / \bar{\lambda}$, we take $\lambda_{\text {eff }}=0$.
} 
$\lambda_{\text {eff }}=\lambda-\bar{\lambda} f^{2}$,

which decrease with melting for increasing $f$.

It is easy to show that

$\varepsilon_{i i}^{e}=\frac{n}{\lambda_{e f f}+\mu_{\text {eff }}}$,

$\left(\varepsilon_{i j}^{e}\right)^{2}=\frac{1}{2}\left[\left(\frac{\tau}{\mu_{e f f}}\right)^{2}+\left(\varepsilon_{i i}^{e}\right)^{2}\right]$,

where $n, \tau$ are normal and shear components of the stresses acting on lubricant from the side of rubbing surfaces. ${ }^{2}$ Relationships (9), (10) relate components of tensors and their invariants of the linear theory of elasticity [38].

Let us write down the kinetic equation for non-equilibrium parameter $f$ in the form of the Landau-Khalatnikov equation:

$\tau_{f} \dot{f}=-\frac{\partial \Phi}{\partial f}$,

where relaxation time $\tau_{f}$ is introduced. In the explicit form it is written as

$\tau_{f} \frac{\partial f}{\partial t}=-c \nabla^{2} f-2 \varphi_{0} f+\varphi_{1} f^{3}-\varphi_{2} f^{5}-\frac{2 n^{2}(\bar{\lambda}+\bar{\mu}) f}{\left(\lambda_{e f f}+\mu_{\text {eff }}\right)^{2}}$,

with the last term showing the dependence of (9) and (10) on $f$.

We derive the equation connecting the relative velocity of shear for rubbing surfaces $V_{i j}$ and the elastic strains arising in the lubricant $\varepsilon_{i j}^{e}$. For this purpose we use the Debye approximation relating the elastic strain and the plastic one $\varepsilon_{i j}^{p l}[5]$ :

$\dot{\varepsilon}_{i j}^{p l}=\frac{\varepsilon_{i j}^{e}}{\tau_{\varepsilon}}$,

where $\tau_{\varepsilon}$ is the Maxwell relaxation time of internal stress. The total strain in a layer is defined as

$\varepsilon_{i j}=\varepsilon_{i j}^{e}+\varepsilon_{i j}^{p l}$.

This strain fixes the velocity of motion of the top block according to equation [40]:

$V_{i j}=h \dot{\varepsilon}_{i j}=h\left(\dot{\varepsilon}_{i j}^{e}+\dot{\varepsilon}_{i j}^{p l}\right)$,

where $h$ is the thickness of lubricant film. The last three relationships give the expression for elastic components of shear strain [35]:

$\tau_{\varepsilon} \dot{\varepsilon}_{i j}^{e}=-\varepsilon_{i j}^{e}+\frac{V_{i j} \tau_{\varepsilon}}{h}$.

Further, for simplicity, a homogeneous system is considered, and in Eqs. (1) and (12) we put $\nabla \equiv 0$.

\section{Friction force}

In view of definitions (3), (6)-(10) the set of kinetic equations (12) and (16) is closed, it can be used for studying the kinetics of lubricant melting. In this section we consider the stationary modes of friction resulting from system evolution. According to (16), in the course of time, the stationary value of elastic component of shear strain is set in

$\varepsilon_{i j 0}^{e}=\frac{V_{i j} \tau_{\varepsilon}}{h}$.

For finding the stationary states of all quantities it is necessary to solve numerically the evolution equation (12), using (3), (6)-(10) and determining the value of strain from (17).

\footnotetext{
${ }^{2}$ Shear stress $\tau$ is defined from expression (6) for $i \neq j$, i.e. $\delta_{i j}=0$. When $\mu_{\text {eff }}=0$ the term $\tau / \mu_{\text {eff }}$ in (10), should be substituted by $2 \varepsilon_{i j}^{e}$, according to (6).
}

The dependences of friction force on velocity of shear, thickness of lubricant layers, and load on the rubbing surfaces are often determined experimentally [1-4,24-26]. Let us analyze how the lubricant temperature and velocity of shear affect the friction force.

In the course of friction, both elastic $\sigma_{i j}^{e}$ and viscous $\sigma_{i j}^{v i s c}$ stresses arise in the lubricant. The total stress in a layer is the sum of these two components

$\sigma_{i j}=\sigma_{i j}^{e}+\sigma_{i j}^{v i s c}$.

The total friction force is defined in a standard way:

$F_{i j}=\sigma_{i j} A$,

where $A$ is the area of contacting surfaces. Viscous stresses in a layer are given by the formula [40]:

$\sigma_{i j}^{v i s c}=\frac{\eta_{e f f} V_{i j}}{h}$,

where $\eta_{\text {eff }}$ is the effective viscosity determined only experimentally, and in the boundary mode [40]

$\eta_{\text {eff }}=k\left(\dot{\varepsilon}_{i j}\right)^{\gamma}$.

Here the proportionality factor $k\left(\mathrm{~Pa} \mathrm{~s}^{\gamma+1}\right)$ is introduced. According to (21), for the pseudoplastic lubricants the coefficient $\gamma<0$, the dilatant lubricants are characterized by the $\gamma>0$. And in the case when lubricants are Newtonian liquids $\gamma=0$ because in accordance with (21), the viscosity does not depend on velocity. In view of (15), (21) the expression for viscous stresses (20) is written in the form

$\sigma_{i j}^{v i s c}=k\left(\frac{V_{i j}}{h}\right)^{\gamma+1}$.

After substitution of (18) and (22) in (19) we have the required expression for the friction force $[35]^{3}$ :

$F_{i j}=\left[\sigma_{i j}^{e}+k \cdot \operatorname{sgn}\left(V_{i j}\right)\left(\frac{\left|V_{i j}\right|}{h}\right)^{\gamma+1}\right] A$,

where $\sigma_{i j}^{e}$ is defined by formula (6) for $i \neq j$.

In experiments, the atomically smooth surfaces of mica are often taken for the friction surfaces and lubricants are the quasispherical molecules of octamethylcyclotetrasiloxane (OMCTS) and linear chain molecules of tetradecane or hexadecane $[2,3,26]$. The experimental conditions are as follows: the thickness of lubricant $h \sim 10^{-9} \mathrm{~m}$, the contact area $A \sim 3 \times 10^{-9} \mathrm{~m}^{2}$, the load on the top surface of friction $L=(2-60) \times 10^{-3} \mathrm{~N}$ that corresponds to normal stresses $n=-L / A=-(6.67-200) \times 10^{5} \mathrm{~Pa}$. The friction force is $F \sim(2-40) \times 10^{-3} \mathrm{~N}$. In the mentioned experiments it has been found that the lubricant melts at a temperature above the critical value $T>T_{c 0} \sim 300 \mathrm{~K}$, or at a velocity of shear $V>V_{c 0} \sim 400 \mathrm{~nm} / \mathrm{s}$. These values may much differ depending on lubricant and geometry of the experiment.

According to experimental data, the following values of theoretical constants are chosen for the considered model [35]: $\Phi_{0}^{*}=20 \mathrm{~J} / \mathrm{m}^{3}$, $\lambda=2 \times 10^{11} \mathrm{~Pa}, \bar{\lambda}=10^{8} \mathrm{~Pa}, \quad \mu=4.1 \times 10^{11} \mathrm{~Pa}, \bar{\mu}=4 \times 10^{11} \mathrm{~Pa}$, $\varphi_{0}^{*}=185 \mathrm{~J} / \mathrm{m}^{3}, \varphi_{1}=570 \mathrm{~J} / \mathrm{m}^{3}, \varphi_{2}=3200 \mathrm{~J} / \mathrm{m}^{3}, \alpha=0.6 \mathrm{~J} \mathrm{~K}{ }^{-1} / \mathrm{m}^{3}$, $h=10^{-9} \mathrm{~m}, \tau_{f}=1$ Pa s, $\tau_{\varepsilon}=10^{-8} \mathrm{~s}$. Note that the relaxation time of excess volume $\tau_{f}$ has the dimension of viscosity. In fact, this means that the time of reaching the stationary friction mode increases with the growth of the effective viscosity of the lubricant.

Fig. 1a illustrates how the friction force decreases with lubricant temperature increase. Let us analyze the curve 1 in detail. At first, with temperature increase to point of phase transition at $T<T_{c 0}$ the excess volume is equal to zero, therefore, the effective shear modulus $2 \mu_{\text {eff }}$ (7) takes constant value. Since the shear velocity

\footnotetext{
${ }^{3}$ Here the sign function $\operatorname{sgn}(x)$ and absolute value of shear velocity $\left|V_{i j}\right|$ are introduced, since $V_{i j}$ can take negative values too.
} 
a

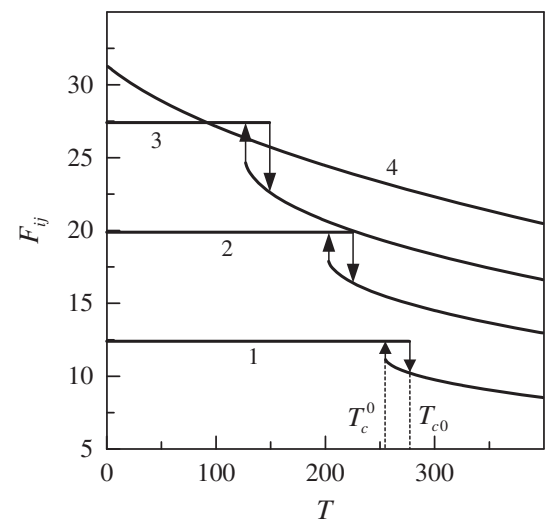

b

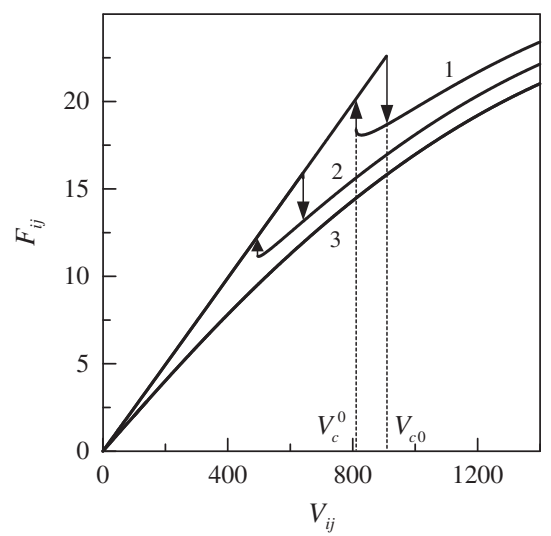

Fig. 1. Dependence of the stationary value of the total friction force $F_{i j}(\mathrm{mN})(23)$ on the temperature of lubricant $T(\mathrm{~K})$ and the velocity of $\operatorname{shear} V_{i j}(\mathrm{~nm} / \mathrm{s})$ for $\gamma=2 / 3, k=1 \mathrm{~Pa} \mathrm{~s}^{5 / 3}$, $A=3 \times 10^{-9} \mathrm{~m}^{2}, n=-7 \times 10^{5} \mathrm{~Pa}$ : (a) curves $1-4$ correspond to the constant values of velocities of shear $V_{i j}=500,800,1100,1550 \mathrm{~nm} / \mathrm{s}$; (b) curves $1-3$ correspond to the fixed values of temperature $T=200,255,310 \mathrm{~K}$.

is invariable, in accordance with (17), the constant value of strain is realized, leading to constant shear stress (6). Thus, the both components in (23) do not change with temperature increase to a point of phase transition, and the friction force is constant. When the temperature becomes higher than the critical value $T>T_{c 0}$, the value of excess volume $f^{2}$ increases abruptly, and the lubricant melts and there is a sharp decrease in the total friction force.

If after melting the temperature is increased, the excess volume increases resulting in the reduction of the shear modulus and, as a consequence, in a smaller value of the total friction force. With temperature fall the lubricant solidifies when $T=T_{c}^{0}$. Thus, the dependence is of hysteretic character that corresponds to the firstorder phase transitions. With the increase in velocity of shear the lubricant melts at a smaller value of temperature. When the velocity exceeds the critical value, the lubricant is always liquidlike independent of temperature (curve 4), and the friction force decreases with temperature increase owing to the decrease in the shear modulus (the lubricant becomes more liquid).

Thus, at small temperatures $\left(T<T_{c}^{0}\right)$ there is one zero minimum of the potential $\Phi(f)$ (solid-like lubricant). In the range of temperatures $T_{c}^{0}<T<T_{c 0}$, together with the specified minimum, two symmetric non-zero minima of $\Phi(f)$ coexist, ${ }^{4}$ however, the system cannot pass to the state corresponding to those minima since they are separated by maxima with the zero minimum. At further increase in temperature $T>T_{c 0}$ the separating maxima disappear, and according to the mechanism of the first-order phase transition, the lubricant passes to the state corresponding to the minimum of potential with $f \neq 0$, i.e. it melts. If now the temperature is decreased, there appears a zero minimum and again the system cannot pass to the respective state because of the presence of separating maxima. With their disappearance at $T=T_{c}^{0}$ the lubricant solidifies abruptly.

Fig. 1b shows a somewhat different behavior. Here, in accordance with (23), at small shear velocities the lubricant is solid-like, and since $f=0$ the value of shear modulus is maximal. In such a mode an increase in the velocity increases the friction force (23) due to the increasing viscous and elastic components of stresses. According to the Hooke law (6), the reason for elastic stress increasing with growth of shear velocity is the increase in elastic strain (17). At $V>V_{c 0}$ the lubricant melts, and elastic shear stresses (6) decrease sharply and the total friction force decreases in a jumplike manner. With further increase in the velocity, the $F_{i j}$ increases owing to viscous and elastic components of the friction force, which

${ }^{4}$ Since $f^{2}$ is an observable quantity, these minima are equivalent with respect to friction mode.

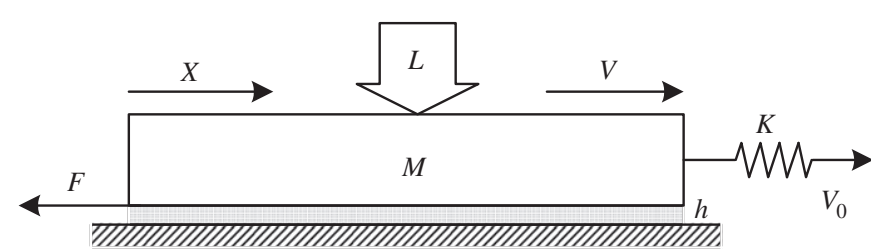

Fig. 2. The scheme of tribological system.

are always increasing with the velocity. According to curve 3 , in the liquid-like state the friction force (23) increase due to the increase in the velocity. With temperature increase the lubricant melts at lower shear velocities. Note that the results of Fig. 1b qualitatively coincide with the new friction map for a boundary mode proposed in work [40] as a result of generalization of experimental data. At present, the dependences of friction force on temperature similar to those presented in Fig. 1a are not measured experimentally.

\section{Stick-slip mode}

The dependences of Fig. 1 have been obtained for the fixed shear velocity of the top rubbing surface. However, the dynamic characteristics of real tribological systems are defined by both the friction force, shown in the figure, and the properties of system as a whole. In particular, according to experiments, in the hysteresis region of the dependence in Fig. 1, the interrupted mode of friction (stick-slip) $[2,3,6,8-10,26,37,40]$ can be realized. The present work is devoted to the definition of its features. A typical scheme of tribological system is presented in Fig. 2. Here, the spring of rigidity $K$ is connected to the block of mass $M$ to which additional normal load $L$ is applied. The block is located on a smooth surface from which it is separated by a layer of lubricant of thickness $h$. The free end of the spring is brought in motion with a constant velocity $V_{0}$. Block motion initiates friction force $F(23)$ that resists its displacement. Generally, for ultrathin layers of lubricants in a mode of boundary friction the velocities of block $V$ and spring $V_{0}$ do not coincide because of the oscillating character of force $F$ leading to interrupted motion of the block. Such mode resembles dry friction without a lubricant.

The equation of motion of the top block looks like $[2,3,5,6]^{5}$

$M \ddot{X}=K \Delta X-F$.

${ }^{5}$ Further, for convenience we omit lower tensor designations since shear in one direction is considered. 
Here $\Delta X$ is the value of extension of a spring which can be defined as

$\Delta X=\int_{0}^{t} V_{0} \mathrm{~d} t^{\prime}-X$,

where $t=t^{\prime}$ is the time of movement of free end of the spring. In the case, when the value of $V_{0}$ is not changed in time, Eq. (25) takes the form

$\Delta X=V_{0} t-X$.

To calculate the time evolution of the friction force, Eqs. (24) and (25) are solved together with (12) and (16), and the friction force is defined from (23). However, the relaxation time of strain $\tau_{\varepsilon}$, may be put small compared with that of excess volume $\tau_{f}$ owing to lubricant's thinness [35]. Therefore, within the limits of approximation $\tau_{f} \gg \tau_{\varepsilon}$, the two Eqs. (24) and (12) are solved jointly and the strain is defined from (17).

The result of solution of the specified equations is shown in Fig. 3. According to the figure, at first, the friction force monotonously increases, since the lubricant is solid-like, and shear velocity $V$ increases. When the velocity exceeds critical value $V_{c 0}$, the lubricant melts and the friction force decreases, the shear velocity of the rubbing block increases, and it quickly moves to a large distance. As a result, the tension of spring and the velocity of shear decrease. When the velocity becomes less than that for the lubricant to be maintained in liquid-like state, it solidifies, and the friction force starts again increasing. The described process is periodically recurrent. It is worth noting that the velocity, with which the lubricant solidifies, does not coincide with a similar velocity shown

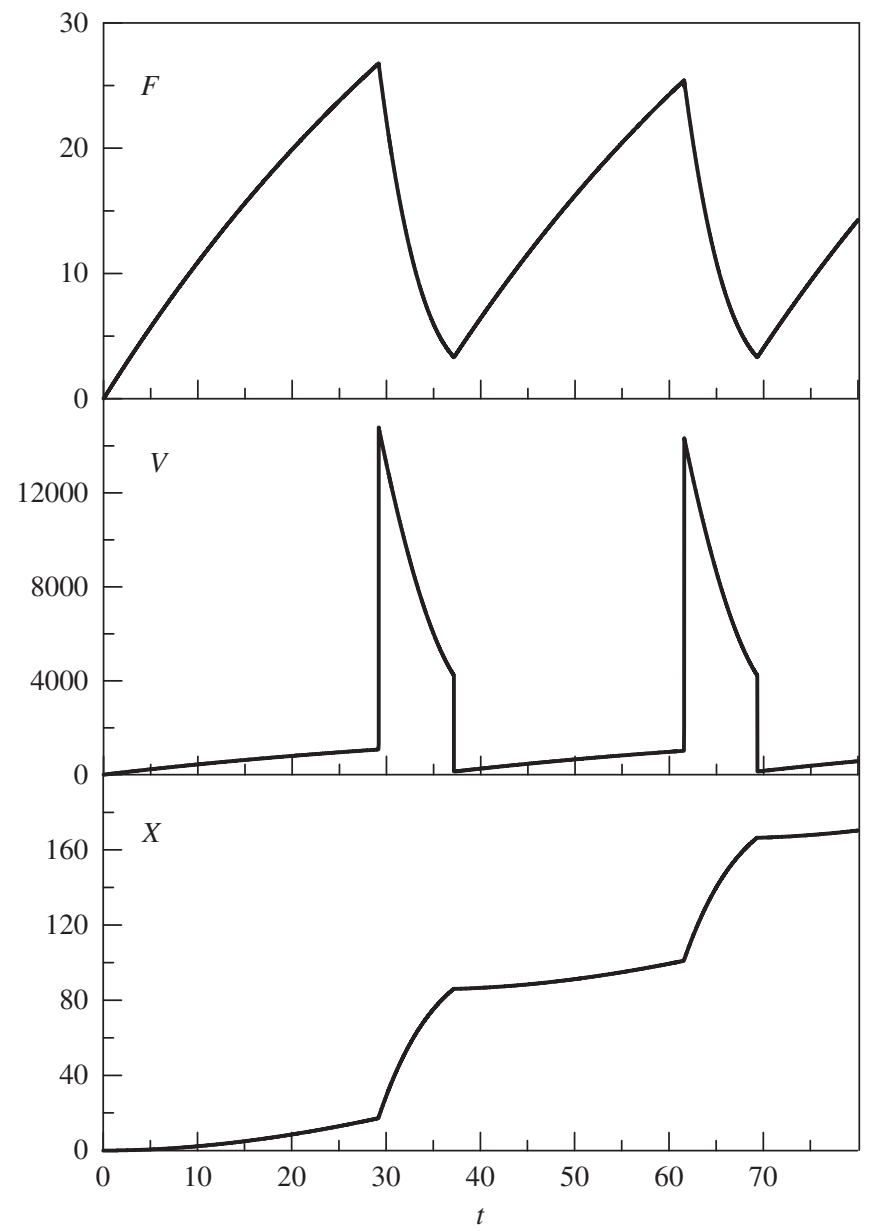

Fig. 3. Dependence of the friction force $F(\mathrm{mN})$, the shear velocity of rubbing surface $V(\mathrm{~nm} / \mathrm{s})$, and its coordinate $X(\mu \mathrm{m})$ on the time $t(\mathrm{~s})$ for parameters of Fig. 1, $M=0.4 \mathrm{~kg}, K=480 \mathrm{~N} / \mathrm{m}, T=250 \mathrm{~K}$, and $V_{0}=2500 \mathrm{~nm} / \mathrm{s}$. in Fig. 1. This is because of the sharp increase in the velocity of shear $V$ at melting and corresponding increase in excess volume $f^{2}$. According to (7), in this case the shear modulus becomes lower than zero, but it need be considered zero and this deforms the potential form (1). Thus, in the presence of elastic strain (17), according to (6), the elastic stresses in the lubricant are equal to zero. As a result, the friction force decreases, and the lubricant flows.

In Fig. 4, there are dependences of the total friction force $F(23)$, the excess volume $f^{2}$, and the elastic component of the shear stress $\sigma_{i j}^{e}(6)$ on the time under $V_{0}$ increase. Initially, the movement of the top sheared block $\left(V_{0}=V_{01}\right)$ leads to the increase in friction force at zero $f^{2}$. When the elastic shear stresses reach the critical value, the shear melting of lubricant is initiated by the first-order phase transition mechanism. Now, parameter $f^{2}$ increases abruptly, and elastic stresses become equal to zero. And again, there follows the lubricant solidification since the relative shear velocity of the friction surfaces decreases (see Fig. 3). When it solidifies completely, the elastic stresses appear there. And again, subsequent increase in the stresses leads to the increase in parameter $f^{2}$ till the critical value needed for melting, and the process is repeated. As a result, the periodic interrupted (stick-slip) mode of melting/ solidification becomes steady. With velocity increase to $V_{0}=V_{02}$ the frequency of stick-slip peaks increases because at this velocity the critical value of stresses is reached more rapidly. Accordingly,

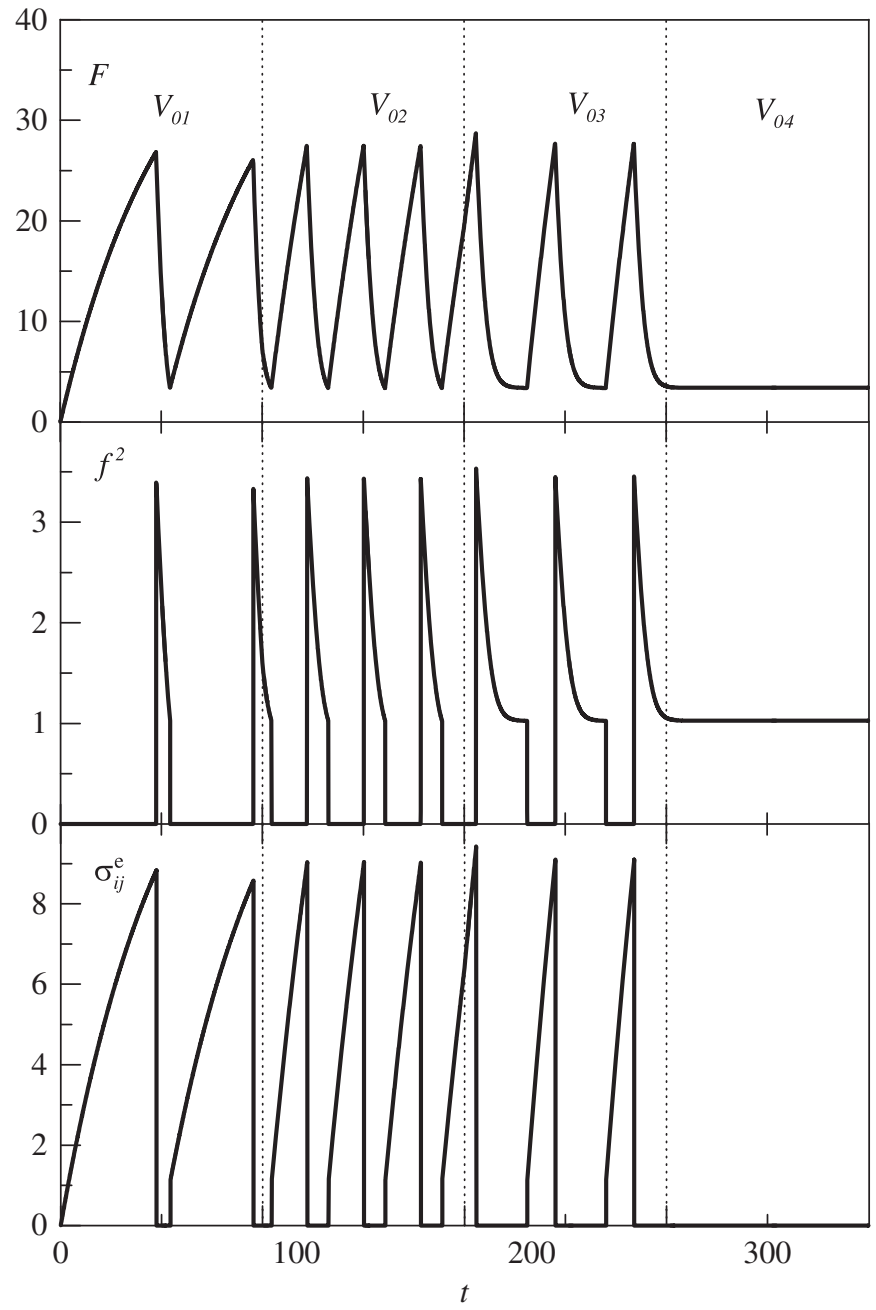

Fig. 4. Dependence of the friction force $F(\mathrm{mN})$, the excess volume $f^{2}$, and the elastic components of shear stress $\sigma_{i j}^{e}(\mathrm{MPa})$ on the time $t(\mathrm{~s})$ for parameters of Fig. 3, normal stress $n=-30 \times 10^{5} \mathrm{~Pa}$, and velocities of shear $V_{01}=1800 \mathrm{~nm} / \mathrm{s}, V_{02}=3500 \mathrm{~nm} / \mathrm{s}$, $V_{03}=4286 \mathrm{~nm} / \mathrm{s}, V_{04}=4290 \mathrm{~nm} / \mathrm{s}$. 
the lubricant melts more rapidly and in the same interval of time the system succeeds in making a greater quantity of melting/ solidification transitions. With a higher increase in velocity $V_{0}=V_{03}$, the frequency of stick-slip peaks decreases again due to the appearance of long kinetic sections $F=$ const in the $F(t)$ dependence. Note that in this mode, at melting, the excess volume $f^{2}$ initially sharply increases owing to rapid increase in shear velocity of the top rubbing block $V$. A smaller value of excess volume $f^{2}$ reached after abrupt initial slip of the rubbing surface at the expense of released mechanical potential energy of compressed spring corresponds to the stationary kinetic section. With further increase in velocity $V_{0}=V_{04}$, the interrupted mode disappears, and the kinetic mode of liquid-like lubricant friction is reached. It is characterized by non-zero value of excess volume $f^{2}$ and elastic stresses $\sigma_{i j}^{e}=0$. Thus, with the increase in velocity, the frequency of stick-slip peaks first increases, then it decreases due to the appearance of long kinetic sections, and when value of velocity exceeds $V_{03}$ the stick-slip mode disappears. The described behavior is in a good agreement with experimental data [2,3].

The influence of the external pressure, applied perpendicularly to the friction surfaces, on character of lubricant melting is often studied experimentally $[2,3,37]$. Such experiments show that pressure effect on the parameters of tribological systems is not trivial. For example, for a lubricant consisting of chain molecules of hexadecane the critical velocity of shear decreases with pressure growth, and on the contrary for spherical molecules of OMCTS it increases [2,3]. Pressure influences the frequency and amplitude of stick-slip transitions too $[2,3]$. Within the limits of our model, according to Eq. (12), the increase in load on the friction surface reduces the excess volume that should promote lubricant solidification.

In Fig. 5, the time dependence of friction force is shown at various values of pressure acting to compress friction surfaces. At a temperature below the critical value (the top panel) the interrupted mode of friction is realized. And with pressure growth the amplitude of stick-slip transitions and values of kinetic and static friction force increase; as to the frequency of transitions, it decreases. At a pressure, corresponding to normal stress $n=n_{4}$, the stick-slip mode is not realized. However, the kinetic mode,

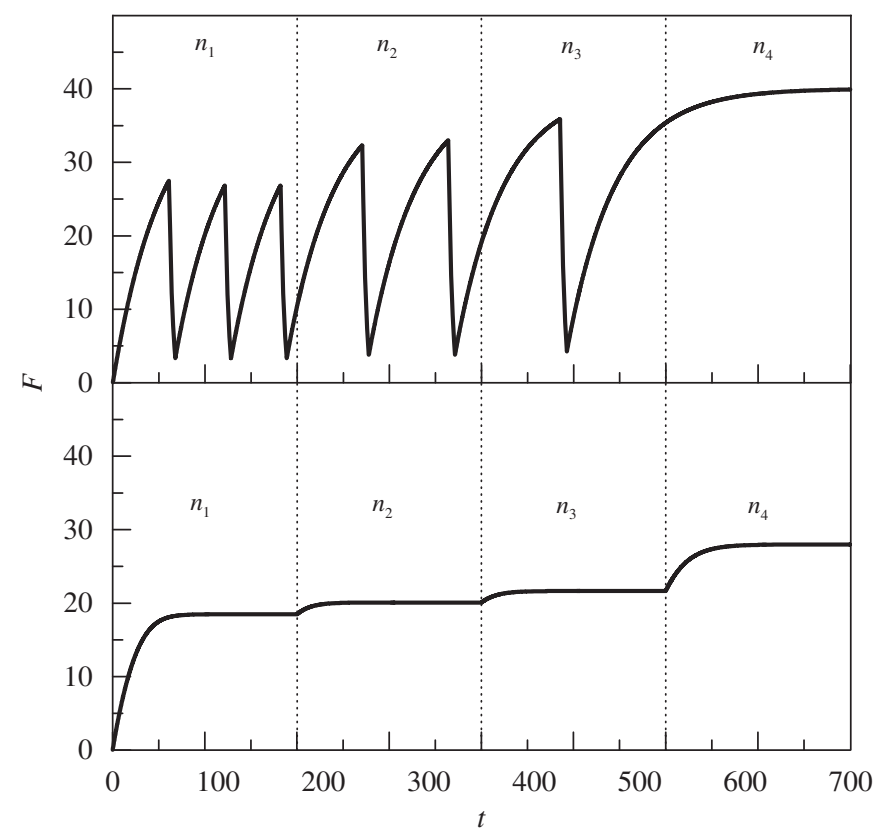

Fig. 5. Dependence of the friction force $F(\mathrm{mN})$ on the time $t(\mathrm{~s})$ for parameters of Fig. 3 , shear velocity $V_{0}=1600 \mathrm{~nm} / \mathrm{s}$, and external normal load $n_{1}=-7 \times 10^{5} \mathrm{~Pa}$, $n_{2}=-70 \times 10^{5} \mathrm{~Pa}, n_{3}=-100 \times 10^{5} \mathrm{~Pa}, n_{4}=-180 \times 10^{5} \mathrm{~Pa}$. The top panel corresponds to the temperature $T=235 \mathrm{~K}$, the bottom one to $T=540 \mathrm{~K}$.

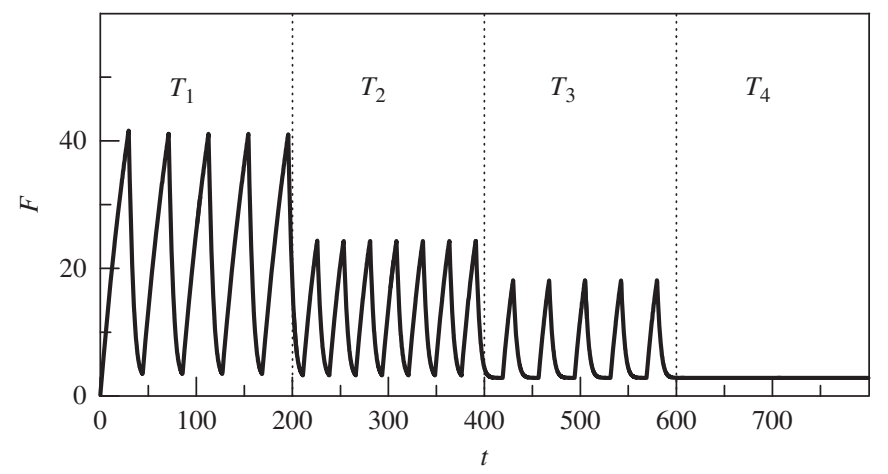

Fig. 6. Dependence of the friction force $F(\mathrm{mN})$ on the time $t(\mathrm{~s})$ for parameters of Fig. 3, velocity $V_{0}=3850 \mathrm{~nm}$, and lubricant temperatures $T_{1}=150 \mathrm{~K}, T_{2}=300 \mathrm{~K}$, $T_{3}=650 \mathrm{~K}, T_{4}=800 \mathrm{~K}$.

corresponding to lubricant melting, does not set in and the lubricant solidifies because of walls squeezing. As a result, the lubricant cannot melt, the friction force $F$ is high, it corresponds to solidlike lubricant and zero value of excess volume $f^{2}$, as the squeezing of walls promotes the formation of long-range order of atoms in the lubricant. In the bottom figure panel, the dependence is for the elevated temperature $T$. Here, we have the kinetic mode of friction corresponding to a small value of the friction force and to non-zero excess volume $f^{2}$. With pressure growth the stick-slip mode is expected, and with a higher loading the full solidification of the lubricant is to be observed the same as in the top panel of the figure for $n=n_{4}$. Thus, three modes of friction have been revealed: (1) the kinetic mode in which the lubricant is always liquid-like; (2) the interrupted mode corresponding to periodic melting/ solidification; (3) the mode of dry friction characterized by larger value of the friction force and solid-like lubricant structure. Similar modes are found also within the framework of stochastic model [8].

In Fig. 6, the dependence of friction force on lubricant temperature is shown. Temperature rise leads to the decrease in the amplitude of friction force oscillations and the change of the frequency of phase transitions. For $T=T_{4}$ the mode of sliding arises characterized by constant values of kinetic friction force and shear velocity of the rubbing block. Thus, the temperature growth promotes lubricant melting. We are not aware of experiments with a similar study of the temperature effect and the given dependence is, therefore, predicting.

\section{Conclusion}

The proposed theory allows to describe the effects observed at the melting of ultrathin lubricant film in a boundary friction mode. The consistent consideration of thermodynamic and shear melting has been carried out. Dependences of the friction force on the shear velocity and the temperature, as well as on the pressure upon surfaces are analyzed. At high lubricant temperature the shear melting is realized at a smaller value of shear velocity (shear stress), and with a still higher increase in temperature the lubricant melts even at zero velocity of shear. The model takes the effect of temperature, shear melting, and pressure into consideration. These are the major factors investigated experimentally.

Within the framework of the proposed theory, a simple tribological system has been studied, and the time dependences of the friction force are obtained for the increasing shear velocity, pressure, and temperature. It is shown that in the system, in a wide range of parameters, the experimentally observable intermittent mode of friction is realized. The obtained results qualitatively coincide with the known experimental data. Since the model is quantitative, it may be modified to describe concrete experiments. 


\section{Acknowledgment}

The work has been done under financial support of the Fundamental Researches State Fund of Ukraine (Grant $\Phi$ 28/443-2009).

\section{References}

[1] Persson BNJ. Sliding friction. Physical principles and applications. New York: Springer-Verlag; 2000.

[2] Yoshizawa H, Chen Y-L, Israelachvili J. Fundamental mechanisms of interfacia friction. 1. Relation between adhesion and friction. J Phys Chem 1993;97: 4128-40.

[3] Yoshizawa H, Israelachvili J. Fundamental mechanisms of interfacial friction. 2. Stick-slip friction of spherical and chain molecules. J Phys Chem 1993;97: 11300-13.

[4] Smith ED, Robbins MO, Cieplak M. Friction on adsorbed monolayers. Phys Rev B 1996;54:8252-60.

[5] Popov VL. Thermodynamics and kinetics of shear-induced melting of a thin lubrication film confined between solids. Tech Phys 2001;46:605-15.

[6] Carlson JM, Batista AA. Constitutive relation for the friction between lubricated surfaces. Phys Rev E 1996;53:4153-65.

[7] Khomenko AV, Yushchenko OV. Solid-liquid transition of ultrathin lubricant film. Phys Rev E 2003;68:036110-6.

[8] Filippov AE, Klafter J, Urbakh M. Friction through dynamical formation and rupture of molecular bonds. Phys Rev Lett 2004;92:135503-4.

[9] Tshiprut Z, Filippov AE, Urbakh M. Tuning diffusion and friction in microscopic contacts by mechanical excitations. Phys Rev Lett 2005;95:016101-4.

[10] Braun OM, Naumovets AG. Nanotribology: microscopic mechanisms of friction. Surf Sci Rep 2006;60:79-158.

[11] Khomenko AV, Prodanov NV. Molecular dynamics simulations of ultrathin water film confined between flat diamond plates. Cond Matt Phys 2008;11: 615-26.

[12] Khomenko AV, Prodanov NV. Molecular dynamics of cleavage and flake formation during the interaction of a graphite surface with a rigid nanoasperity. Carbon 2010;48:1234-43.

[13] Prodanov NV, Khomenko AV. Computational investigation of the temperature influence on the cleavage of a graphite surface. Surf Sci 2010;604:730-40.

[14] Brener EA, Marchenko VI. Frictional shear cracks. JETP Lett 2002;76:211-4.

[15] Horn RG, Smith DT, Haller W. Surface forces and viscosity of water measured between silica sheets. Chem Phys Lett 1989;162:404-8.

[16] Khomenko AV, Lyashenko IA. Stochastic theory of ultrathin lubricant film melting in stick-slip regime. Tech Phys 2005;50:1408-16.

[17] Khomenko AV, Lyashenko IA. Melting of ultrathin lubricant film due to dissipative heating of friction surfaces. Tech Phys 2007;52:1239-43.

[18] Khomenko AV, Lyashenko IA, Borisyuk VN. Multifractal analysis of stress time series during ultrathin lubricant film melting. Fluct Noise Lett 2010;9:19-35.
[19] Khomenko AV, Lyashenko IA, Borisyuk VN. Self-similar phase dynamics of boundary friction. Ukr J Phys 2009:54:1139-48.

[20] Khomenko AV, Lyashenko IA. Phase dynamics and kinetics of thin lubricant film driven by correlated temperature fluctuations. Fluct Noise Lett 2007;7:L111-33.

[21] Carpinteri A, Spagnoli A, Vantadori S. Size effect in S-N curves: a fracta approach to finite-life fatigue strength. Int J Fatigue 2009;31:927-33.

[22] Carpinteri A, Spagnoli A, Vantadori S. A multifractal analysis of fatigue crack growth and its application to concrete. Eng Fract Mech 2010;77:974-84

[23] Khomenko AV, Lyashenko IA. Temperature dependence effect of viscosity on ultrathin lubricant film melting. Condens Matter Phys 2006;9:695-702.

[24] Demirel AL, Granick S. Transition from static to kinetic friction in a model lubricating system. J Chem Phys 1998;109:6889-97.

[25] Reiter G, Demirel AL, Peanasky J, Cai LL, Granick S. Stick to slip transition and adhesion of lubricated surfaces in moving contact. J Chem Phys 1994;101: 2606-15.

[26] Israelachvili J. Adhesion forces between surfaces in liquids and condensable vapours. Surf Sci Rep 1992;14:109-59.

[27] Khomenko AV, Lyashenko IA. Hysteresis phenomena during melting of an ultrathin lubricant film. Phys Sol State 2007;49:936-40.

[28] Khomenko AV, Lyashenko IA. Hysteresis phenomena at ultrathin lubricant film melting in the case of first-order phase transition. Phys Lett A 2007;366: 165-73.

[29] Khomenko AV, Lyashenko IA. Periodic intermittent regime of a boundary flow. Tech Phys 2010;55:26-32.

[30] Braun OM, Röder J. Transition from stick-slip to smooth sliding: an earthquake like model. Phys Rev Lett 2002;88:096102-4.

[31] Aranson IS, Tsimring LS, Vinokur VM. Stick-slip friction and nucleation dynamics of ultrathin liquid films. Phys Rev B 2002;65:125402-7.

[32] Lemaître A. Rearrangements and dilatancy for sheared dense materials. Phys Rev Lett 2002;89:195503-4.

[33] Metlov LS. Formation of the internal structure of solids under severe load. Phys Rev E 2010;81:051121-9.

[34] Metlov LS. Thermodynamics of nonequilibrium processes in application to severe plastic deformation. Bull Russ Acad Sci Phys 2008;72:1283-7.

[35] Lyashenko IA, Khomenko AV, Metlov LS. Phenomenological theory for the melting of a thin lubricant film between two atomically smooth solid surfaces. Tech Phys 2010;55:1193-9.

[36] Thompson PA, Grest GS, Robbins MO. Phase transitions and universal dynamics in confined films. Phys Rev Lett 1992;68:3448-51.

[37] Gee ML, McGuiggan PM, Israelachvili JN. Liquid to solid like transitions of molecularly thin films under shear. J Chem Phys 1990;93:1895-906.

[38] Kachanov LM. Foundations of the theory of plasticity. Amsterdam: NorthHolland; 1971.

[39] Landau LD, Lifshitz EM. Course of theoretical physics, vol. 7. Theory of elasticity. 3rd ed. New York: Pergamon Press; 1986.

[40] Luengo G, Israelachvili J, Granick S. Generalized effects in confined fluids: new friction map for boundary lubrication. Wear 1996;200:328-35. 